\title{
UNSTEADY MHD CHEMICALLY REACTING FLUID THROUGH A POROUS MEDIUM BOUNDED BY A NON-ISOTHERMAL IMPULSIVELY-STARTED VERTICAL PLATE: A NUMERICAL TECHNIQUE
}

\author{
Sahin Ahmed ${ }^{1}$ and Karabi Kalita ${ }^{2}$
}

${ }^{1}$ Heat Transfer and Fluid Mechanics Research, Department of Mathematics \& Computing, Rajiv Gandhi University, Rono Hills, Itanagar 783101, Arunachal Pradesh, India, Email: heat_mass@yahoo.in

${ }^{2}$ Department of Mathematics, Gauhati University, Guwahati 781014, Assam, India, Email: kkarabi88@gmail.com

\begin{abstract}
:
A numerical modeling on MHD transient mass transfer by free convection flow of a viscous, incompressible, electrically-conducting, and Newtonian fluid through a porous medium bounded by an impulsively-started semi-infinite vertical plate in the presence of thermal radiation and chemical reaction of first order has been analyzed. The fluid is assumed optically thin gray gas, absorbingemitting radiation, but a non-scattering medium. The dimensionless governing coupled, non-linear boundary layer partial differential equations are solved by an efficient, accurate, and extensively validated and unconditionally stable finite difference scheme of the Crank-Nicolson type. The effects of the conduction-radiation parameter $\left(R_{a}=0.0,0.5,1.0,3.0,5.0\right)$, chemical reaction $\left(C_{r}=-1.5,-1.0\right.$, $0.0,1.0,1.5)$ and the porosity $(K=0.0,0.5,1.5,3.0,5.0)$ on the velocity, temperature and concentration fields have been studied. The local skin friction, Nusselt number and the Sherwood number are also presented graphically and analyzed. Increasing magnetic parameter serves to decelerate the flow but increased temperature and concentration values. It is found that the velocity is increased considerably with a rise in the porosity parameter $(K)$ whereas the temperature and concentration are found to be reduced with increasing porosity $(K)$. An increase in the porosity parameter $(K)$ is found to escalate the local skin friction $\left(\tau_{x}\right)$, Nusselt number $\left(N u_{x}\right)$ and the Sherwood number $\left(S h_{x}\right)$. Possible applications of the present study include laminar aerodynamics, materials processing and thermo-fluid dynamics.
\end{abstract}

Keywords: Magneto-hydrodynamics (MHD), mass transfer, Crank-Nicolson method, porous media, aerothermal analysis.

\section{NOMENCLATURE}

$\bar{C} \quad$ Species concentration $\left(\mathrm{Kg} \cdot \mathrm{m}^{-3}\right)$

$C_{P} \quad$ Specific heat at constant pressure (J. $\left.\mathrm{kg}^{-1} . \mathrm{K}\right)$

$\bar{C}_{\infty} \quad$ Species concentration in the free stream (Kg. $\mathrm{m}^{-3}$ )

$\bar{C}_{w} \quad$ Species concentration at the surface $\left(\mathrm{Kg} . \mathrm{m}^{-3}\right.$ )

D Chemical molecular diffusivity $\left(\mathrm{m}^{2} \cdot \mathrm{s}^{-1}\right)$

$g \quad$ Acceleration due to gravity $\left(\mathrm{m} \cdot \mathrm{s}^{-2}\right)$

Gr Thermal Grashoff number

$G r_{m} \quad$ Mass Grashoff number

$M \quad$ Hartmann number

$q_{r} \quad$ Radiation heat flux

$E_{C} \quad$ Eckert number

$K \quad$ Permeability parameter

$n \quad$ Surface temperature power law exponent

$m \quad$ Surface concentration power law exponent

$N u_{x} \quad$ Nusselt number

$S h_{x} \quad$ Sherwood number

$\bar{p} \quad$ Pressure $(\mathrm{Pa})$

$\mathrm{Pr} \quad$ Prandtl number

Sc Schmidt number

$\bar{T} \quad$ Temperature (K)

$\bar{T}_{w} \quad$ Fluid temperature at the surface (K)

$\bar{T}_{\infty} \quad$ Fluid temperature in the free stream (K)

$u \quad$ Dimensionless velocity component in $x$ direction $\left(\mathrm{m} . \mathrm{s}^{-1}\right)$

$v \quad$ Dimensionless velocity component in $y$ direction $\left(\mathrm{m} . \mathrm{s}^{-1}\right)$

$u_{0} \quad$ Plate velocity (m. $\mathrm{s}^{-1}$ )

$\bar{a} \quad$ Absorption coefficient

$\bar{\sigma} \quad$ Stefan-Boltzmann constant,

Greek symbols

$\beta \quad$ Coefficient of volume expansion for heat transfer $\left(\mathrm{K}^{-1}\right)$, 


$\begin{array}{ll}\bar{\beta} & \text { Coefficient of volume expansion for mass } \\ & \text { transfer }\left(\mathrm{K}^{-1}\right) \\ \alpha & \text { Thermal diffusivity } \\ \theta & \text { Dimensionless fluid temperature }(\mathrm{K}) \\ \kappa & \text { Thermal conductivity }\left(\mathrm{W} . \mathrm{m}^{-1} \cdot \mathrm{K}^{-1}\right) \\ \mu & \text { Coefficient of viscosity }\left(\mathrm{kg} . \mathrm{m}^{-3}\right) \\ v & \text { Kinematic viscosity }\left(\mathrm{m}^{2} \cdot \mathrm{s}^{-1}\right) \\ \rho & \text { Density }\left(\mathrm{kg} . \mathrm{m}^{-3}\right)\end{array}$

\section{Introduction}

Magneto-hydrodynamic transport phenomena arise in numerous branches of modern chemical engineering. These include crystal magnetic damping control, hydro-magnetic chromatography, conducting flows in tricklebed reactors and enhanced magnetic filtration control. Numerous studies of both an experimental and numerical nature have been communicated regarding magneto-hydrodynamic flow in chemical engineering. In general, in such flows, the Lorentz electromagnetic force arises as a result of the interaction between the magnetic field and the electrical current. This latter is generated hydro-dynamically by the bulk flow of the conducting liquid phase. In porous media applications such as packed beds, to sustain a given flow rate of the electrically conducting liquid in the bed, the pressure drop and the liquid holdup will be increased under magnetohydrodynamic conditions compared with the case of non-conducting fluids. In crystal growth applications in porous media, the external magnetic field imposed has been successfully exploited to suppress unsteady flow and also reduce composition non-uniformity. Recent monographs by Nield and Bejan (1999) and Ingham and Pop (1998) provide an excellent summary of the work on the subject.

In case of large temperature differences between the surface and the ambient causes, the radiation effect may become important in natural convection, as in many engineering processes. The radiation effects have important applications in physics and engineering, particularly in space technology and high temperature processes. But very little is known about the effects of radiation on the boundary layer. Thermal radiation effects on the boundary layer may play important role in controlling heat transfer in polymer processing industry where the quality of the final product depends on the heat controlling factors to some extent. High temperature plasmas, cooling of nuclear reactors, liquid metal fluids, and power generation systems are some important applications of radiative heat transfer. Actually, many processes in new engineering areas occur at high temperatures and knowledge of radiation heat transfer beside the convective heat transfer becomes very important for the design of the pertinent equipment. Nuclear power plants, gas turbines and the various propulsion devices for aircraft, missiles, satellites and space vehicles are examples of such engineering areas. England and Emery (1969) have studied the radiation effects of an optically thin gray gas bounded by a stationary plate. Again Raptis and Perdikis (2003) studied the effects of thermal radiation on moving vertical plate in the presence of mass diffusion. The governing equations were solved by the Laplace transform technique. The thermal radiation effects in a steady free convective flow through a porous medium bounded by a vertical infinite porous plate have been considered by Raptis (1998). Hussain and Pop (2001) studied the radiation effects on stationary free convection of an optically thin dense fluid along an isothermal vertical surface embedded in a porous medium with highly porosity using Brinkman-Forchheimer-Darcy extended model, by means of two different numerical techniques, the Keller-box method and the local non-similarity scheme. Jaiswal and Soundalgekar (2001) obtained an approximate solution to the problem of an unsteady flow past an infinite vertical plate with constant suction and embedded in a porous medium with oscillating plate temperature.

An analysis of the thermal radiation effects on stationary mixed convection from vertical surfaces in saturated porous media for both a hot and a cold surface has been presented by Bakier (2001). The unsteady flow through a highly porous medium in the presence of radiation was studied by Raptis and Perdikis (2004). The effects of radiation and viscous dissipation on the transient natural convection-radiation flow of viscous dissipation fluid along an infinite vertical surface embedded in a porous medium, by means of network simulation method, investigated by Zueco (2008). The effects of radiation and chemical reaction on natural convection flows of a Newtonian fluid along a vertical surface embedded in a porous medium presented by Mahmud and Chamkha (2010). Sahin (2008) investigated the effect of transverse periodic permeability oscillating with time on the heat transfer flow of a viscous incompressible fluid through a highly porous medium bounded by an infinite vertical porous plate, by means of series solution method. Sahin (2010) studied the effect of transverse periodic permeability oscillating with time on the free convective heat transfer flow of a viscous incompressible fluid 
through a highly porous medium bounded by an infinite vertical porous plate subjected to a periodic suction velocity.

Combined heat and mass transfer occurs frequently in nature. It occurs not only due to temperature difference, but also due to concentration difference or the combination of these two. Chemical reactions can be codified as either heterogeneous or homogeneous process. This depends on whether they occur at an interface or as a single volume reaction. Many transport processes exits in nature and in industrial applications in which the simultaneous heat and mass transfer as a result of combined buoyancy effects of thermal diffusion and diffusion of chemical species. In many chemical engineering processes, there does occur the chemical reaction between a foreign mass and the fluid in which the plate is moving. This process takes in numerous industrial applications, e.g. polymer production, manufacturing of ceramics or glassware and food processing. Chamkha (2003) investigated the chemical reaction effects on heat and mass transfer laminar boundary layer flow in the presence of heat generation/absorption effects. Muthucumaraswamy and Kulaivel (2003) presented an analytical solution to the problem of flow past an impulsively started infinite vertical plate in the presence of heat flux and variable mass diffusion, taking into account the presence of a homogeneous chemical reaction of first order. Sahin and Chamkha (2011) analyzed the effects of radiation and chemical reaction on steady mixed convective heat and mass transfer flow of an optically thin gray gas over an infinite vertical porous plate with constant suction in presence of transverse magnetic field, and they found that the velocity is reduced considerably with a rise in the conduction-radiation parameter. The study of heat and mass transfer on the free convective flow of a viscous incompressible fluid past an infinite vertical porous plate in presence of transverse sinusoidal suction velocity and a constant free stream velocity was presented by Sahin (2009). Also, Sahin and Liu (2010) analyzed the effects of mixed convection and mass transfer of three-dimensional oscillatory flow of a viscous incompressible fluid past an infinite vertical porous plate in presence of transverse sinusoidal suction velocity oscillating with time and a constant free stream velocity. The non-linear coupled equations of the works $(2009,2010)$ were solved analytically by employing perturbation technique.

Yang (1992) employed the method of moments to study the Interaction of mixed convection and thermal radiation in gray, absorbing, emitting laminar gas flow in a pipe, showing that radiation depresses the buoyancy effect, whereas heat transfer rate is enhanced with increasing radiation-conduction parameter. Makinde (2005) used a superposition technique and a Rosseland diffusion flux model to study the natural convection heat and mass transfer in a gray, absorbing-emitting fluid along a porous vertical translating plate. Loganathan et al. (2008) investigated first order chemical reaction on flow past an impulsively started semi-infinite vertical plate in the presence of thermal radiation by an implicit finite-difference scheme of the Crank-Nicolson type. The effects of Darcian drag force and radiation-conduction on unsteady two-dimensional magneto-hydrodynamic heat transfer flow of viscous, electrically conducting and Newtonian fluid over a vertical plate adjacent to a Darcian regime in presence of thermal radiation and transversal magnetic field were studied by Sahin et al. (2014). The first-order chemical reaction as well as the magneto-hydrodynamic effects on heat and mass transfer flow past an impulsively started semi-infinite vertical plate in the presence of thermal radiation by an implicit finite-difference scheme was presented by Sahin et al. (2014). Zueco et al. (2014) consider the 2D magnetohydrodynamics of an incompressible micropolar fluid over a stretching sheet when the sheet is stretched in its own plane in a Darcian porous regime. The stretching velocity is assumed to vary linearly with the distance along the sheet. The transformed governing partial differential equations are solved numerically using the Network Simulation Method (NSM) for some values of the physically governing parameters.

The present work investigates numerically porosity and chemical reaction effects on unsteady MHD heat and mass transfer flow past an impulsively-started semi-infinite vertical plate embedded in a porous medium in the presence of thermal radiation by an implicit finite-difference scheme. Such a study has immediate applications in the chemical process engineering. A well-tested, numerically stable Crank-Nicolson finite-difference procedure is employed.

\section{Mathematical Analysis:}

A two-dimensional, transient, laminar, natural convection flow of a viscous incompressible fluid through a porous medium bounded by an impulsively-started semi-infinite vertical plate in the presence of conductionradiation has been considered. It is assumed that a first-order chemical reaction between the diffusing species and the fluid exists. Here, the $x$-axis is taken along the plate in the vertically upward direction and the $y-$ axis is taken normal to the plate. Initially, it is assumed that the plate and the fluid are of the same temperature and 
the temperature of the plate and the concentration level are also raised to $\bar{T}_{w}=\bar{T}_{\infty}+a x^{n}$ and $\bar{C}_{w}=\bar{C}_{\infty}+b x^{m}$. They are maintained at the same level for all time $\bar{t}>0$.

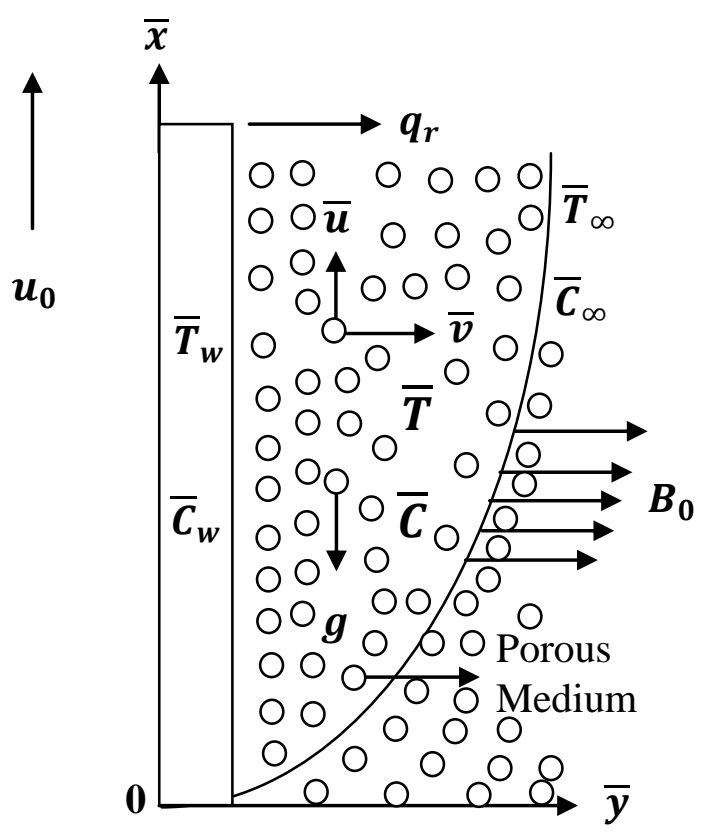

Fig. 1: Physical configuration and coordinate system

Then under the above assumptions, the governing boundary layer equations for free convective MHD flow through porous medium with usual Boussinesq's approximation are as follows:

\section{Equation of Continuity:}

$\frac{\partial \bar{u}}{\partial \bar{x}}+\frac{\partial \bar{v}}{\partial \bar{y}}=0$

\section{Equation of Momentum:}

$\frac{\partial \bar{u}}{\partial \bar{t}}+\bar{u} \frac{\partial \bar{u}}{\partial \bar{x}}+\bar{v} \frac{\partial \bar{u}}{\partial \bar{y}}=g \beta\left(\bar{T}-\bar{T}_{\infty}\right)+g \bar{\beta}\left(\bar{C}-\bar{C}_{\infty}\right)+v \frac{\partial^{2} \bar{u}}{\partial \bar{y}^{2}}-\left(\frac{\sigma B_{0}^{2}}{\rho}+\frac{v}{\bar{K}}\right) \bar{u}$

Equation of Energy:

$\frac{\partial \bar{T}}{\partial \bar{t}}+\bar{u} \frac{\partial \bar{T}}{\partial \bar{x}}+\bar{v} \frac{\partial \bar{T}}{\partial \bar{y}}=\frac{1}{\rho C_{P}}\left(\kappa \frac{\partial^{2} \bar{T}}{\partial \bar{y}^{2}}-\frac{\partial q_{r}}{\partial \bar{y}}\right)$

Equation of mass diffusion:

$\frac{\partial \bar{C}}{\partial \bar{t}}+\bar{u} \frac{\partial \bar{C}}{\partial \bar{x}}+\bar{v} \frac{\partial \bar{C}}{\partial \bar{y}}=D \frac{\partial^{2} \bar{C}}{\partial \bar{y}^{2}}-\bar{C}_{r} \bar{C}$

The initial and boundary conditions are

$\left.\begin{array}{l}\bar{t} \leq 0: \bar{u}=0, \bar{v}=0, \bar{T}=\bar{T}_{\infty}, \bar{C}=\bar{C}_{\infty} \\ \bar{t}>0: \bar{u}=u_{0}, \bar{v}=0, \bar{T}=\bar{T}_{\infty}+a x^{n}, \bar{C}=\bar{C}_{\infty}+b x^{m} \text { at } \bar{y}=0 \\ \bar{u}=0, \bar{T}=\bar{T}_{\infty}, \bar{C}=\bar{C}_{\infty} \text { at } \bar{x}=0 \\ \bar{u} \rightarrow 0, \bar{T} \rightarrow \bar{T}_{\infty}, \bar{C} \rightarrow \bar{C}_{\infty} \text { as } \bar{y} \rightarrow \infty\end{array}\right\}$

For the case of an optically thin gray gas, the local radiant absorption is expressed by 
$\frac{\partial q_{r}}{\partial \bar{y}}=-4 \bar{a} \bar{\sigma}\left(\bar{T}_{\infty}^{4}-\bar{T}^{4}\right)$

We assume that the temperature differences within the flow are sufficiently small such that $\bar{T}^{4}$ may be expressed as a linear function of the temperature. This is accomplished by expanding $\bar{T}^{4}$ in a Taylor series about $\bar{T}_{\infty}$ and neglecting higher-order terms, thus

$\bar{T}^{4} \cong 4 \bar{T}_{\infty}^{3} \bar{T}-3 \bar{T}_{\infty}^{4}$

By using equations (6) and (7), equation (3) reduces to

$\frac{\partial \bar{T}}{\partial \bar{t}}+\bar{u} \frac{\partial \bar{T}}{\partial \bar{x}}+\bar{v} \frac{\partial \bar{T}}{\partial \bar{y}}=\frac{1}{\rho C_{P}}\left[\kappa \frac{\partial^{2} \bar{T}}{\partial \bar{y}^{2}}-16 \bar{a} \sigma \bar{T}_{\infty}^{3}\left(\bar{T}-\bar{T}_{\infty}\right)\right]$

On introducing the following non-dimensional quantities:

$x=\frac{u_{0} \bar{x}}{v}, y=\frac{u_{0} \bar{y}}{v}, u=\frac{\bar{u}}{u_{0}}, v=\frac{\bar{v}}{u_{0}}, t=\frac{\bar{t} u_{0}^{2}}{v}, S c=\frac{v}{D}, \theta=\frac{\bar{T}-\bar{T}_{\infty}}{\bar{T}_{w}-\bar{T}_{\infty}}$

$\left.\begin{array}{l}\phi=\frac{\bar{C}-\bar{C}_{\infty}}{\bar{C}_{w}-\bar{C}_{\infty}}, \operatorname{Pr}=\frac{\rho v C_{P}}{\kappa}, \quad G r=\frac{v g \beta\left(\bar{T}_{w}-\bar{T}_{\infty}\right)}{u_{0}^{3}}, G r_{m}=\frac{v g \bar{\beta}\left(\bar{C}_{w}-\bar{C}_{\infty}\right)}{u_{0}^{3}}, \\ K=\frac{u_{0}^{2} \bar{K}}{v^{2}}, \quad C_{r}=\frac{v \bar{C}_{r}}{u_{0}^{2}}, \quad R_{a}=\frac{64 \bar{a} v^{2} \bar{\sigma} \bar{T}_{\infty}^{3}}{\kappa u_{0}^{2}}, M=\frac{\sigma B_{0}^{2} v}{\rho u_{0}^{2}}\end{array}\right\}$

Equations (1) to (4) are reduced to the following non-dimensional form:

$$
\begin{aligned}
& \frac{\partial u}{\partial x}+\frac{\partial v}{\partial y}=0 \\
& \frac{\partial u}{\partial t}+u \frac{\partial u}{\partial x}+v \frac{\partial u}{\partial y}=G r \theta+G r_{m} \phi+\frac{\partial^{2} u}{\partial y^{2}}-\left(M+K^{-1}\right) u \\
& \frac{\partial \theta}{\partial t}+u \frac{\partial \theta}{\partial x}+v \frac{\partial \theta}{\partial y}=\frac{1}{P r}\left(\frac{\partial^{2} \theta}{\partial y^{2}}-R_{a} \theta\right) \\
& \frac{\partial \phi}{\partial t}+u \frac{\partial \phi}{\partial x}+v \frac{\partial \phi}{\partial y}=\frac{1}{S c} \frac{\partial^{2} \phi}{\partial y^{2}}-C_{r} \phi
\end{aligned}
$$

The corresponding initial and boundary conditions in non-dimensional form are

$t \leq 0: u=0, v=0, \theta=0, \phi=0$

$t>0: u=1, v=0, \theta=x^{n}, \phi=x^{m}$ at $y=0$

$t>0: u=0, \theta=0, \phi=0 \quad$ at $x=0$

$t>0: u \rightarrow 0, \theta \rightarrow 0, \phi \rightarrow 0$ as $y \rightarrow \infty$

The local skin friction (wall shear stress), local Nusselt number (surface heat transfer gradient) and the local Sherwood number (surface species transfer gradient) are given respectively by

$$
\begin{aligned}
& \tau_{x}=-\mu\left(\frac{\partial \bar{u}}{\partial \bar{y}}\right)_{\bar{y}=0}=-\left(\frac{\partial u}{\partial y}\right)_{y=0} \\
& N u_{x}=-\frac{\bar{x}}{\bar{T}_{w}-\bar{T}_{\infty}}\left(\frac{\partial \bar{T}}{\partial \bar{y}}\right)_{\bar{y}=0}=-x\left(\frac{\partial \theta}{\partial y}\right)_{y=0} \\
& S h_{x}=-\frac{\bar{x}}{\bar{C}_{w}-\bar{C}_{\infty}}\left(\frac{\partial \bar{C}}{\partial \bar{y}}\right)_{\bar{y}=0}=-x\left(\frac{\partial \phi}{\partial y}\right)_{y=0}
\end{aligned}
$$




\section{Numerical Techniques:}

In order to solve the unsteady, non-linear coupled equations (10) to (13) under the conditions (14), an implicit finite difference scheme of the Crank-Nicolson type has been employed. The finite difference equations corresponding to Equations (10) to (13) are as follows:

$$
\begin{aligned}
& \frac{\left[u_{i, j}^{n+1}-u_{i-1, j}^{n+1}+u_{i, j}^{n}-u_{i-1, j}^{n}-u_{i-1, j-1}^{n+1}+u_{i, j-1}^{n}-u_{i-1, j-1}^{n}\right]}{4 \Delta x} \\
& +\frac{\left[v_{i, j}^{n+1}-v_{i-1, j}^{n+1}+v_{i, j}^{n}-u_{i, j-1}^{n}\right]}{2 \Delta \mathrm{y}}=0 \\
& \frac{\left[u_{i, j}^{n+1}-u_{i, j}^{n}\right]}{\Delta \mathrm{t}}+u_{i, j}^{n} \frac{\left[u_{i, j}^{n+1}-u_{i-1, j}^{n+1}+u_{i, j}^{n}-u_{i-1, j}^{n}\right]}{2 \Delta x}+v_{i, j}^{n} \frac{\left[u_{i, j+1}^{n+1}-u_{i, j-1}^{n+1}+u_{i, j+1}^{n}-u_{i, j-1}^{n}\right]}{4 \Delta \mathrm{y}} \\
& =\frac{G r\left[\theta_{i, j}^{n+1}+\theta_{i, j}^{n}\right]}{2}+\frac{G r_{m}\left[\phi_{i, j}^{n+1}+\phi_{i, j}^{n}\right]}{2}+\frac{1}{2(\Delta y)^{2}}\left[u_{i, j-1}^{n+1}-2 u_{i, j}^{n+1}+u_{i, j+1}^{n+1}\right. \\
& \left.+u_{i, j-1}^{n}-2 u_{i, j}^{n}+u_{i, j+1}^{n}\right]-\frac{\left[u_{i, j}^{n+1}+u_{i, j}^{n}\right]}{2}\left(M+K^{-1}\right) \\
& \frac{\left[\theta_{i, j}^{n+1}-\theta_{i, j}^{n}\right]}{\Delta \mathrm{t}}+u_{i, j}^{n} \frac{\left[\theta_{i, j}^{n+1}-\theta_{i-1, j}^{n+1}+\theta_{i, j}^{n}-\theta_{i-1, j}^{n}\right]}{2 \Delta x}+v_{i, j}^{n} \frac{\left[\theta_{i, j+1}^{n+1}-\theta_{i, j-1}^{n+1}+\theta_{i, j+1}^{n}-\theta_{i, j-1}^{n}\right]}{4 \Delta \mathrm{y}} \\
& =\frac{\left[\theta_{i, j-1}^{n+1}-2 \theta_{i, j}^{n+1}+\theta_{i, j+1}^{n+1}+\theta_{i, j-1}^{n}-2 \theta_{i, j}^{n}+\theta_{i, j+1}^{n}\right]}{2 \operatorname{Pr}(\Delta \mathrm{y})^{2}}-\frac{R_{a}\left[\theta_{i, j}^{n+1}+\theta_{i, j}^{n}\right]}{2 \operatorname{Pr}} \\
& \frac{\left[\phi_{i, j}^{n+1}-\phi_{i, j}^{n}\right]}{\Delta \mathrm{t}}+u_{i, j}^{n} \frac{\left[\phi_{i, j}^{n+1}-\phi_{i-1, j}^{n+1}+\phi_{i, j}^{n}-\phi_{i-1, j}^{n}\right]}{2 \Delta x} \\
& +v_{i, j}^{n} \frac{\left[\phi_{i, j+1}^{n+1}-\phi_{i, j-1}^{n+1}+\phi_{i, j+1}^{n}-\phi_{i, j-1}^{n}\right]}{4 \Delta \mathrm{y}} \\
& =\frac{\left[\phi_{i, j-1}^{n+1}-2 \phi_{i, j}^{n+1}+\phi_{i, j+1}^{n+1}+\phi_{i, j-1}^{n}-2 \phi_{i, j}^{n}+\phi_{i, j+1}^{n}\right]}{2 S c(\Delta y)^{2}}-\frac{C_{r}\left[\phi_{i, j}^{n+1}+\phi_{i, j}^{n}\right]}{2 S c}
\end{aligned}
$$

The region of integration is considered as a rectangle with sides $x_{\max }(=1)$ and $y_{\max }(=14)$, where $y_{\max }$ corresponds to $y=\infty$ which lies very well outside both the momentum and energy boundary layers. The maximum of $y$ was chosen as 14 after some preliminary investigations so that the last two of the boundary conditions (14) are satisfied with in the tolerance limit $10^{-5}$. After experimenting with a few set of mesh sizes, the mesh sizes have been fixed at the level $\Delta x=0.05, \Delta y=0.25$ with time step $\Delta t=0.01$. In this case, the spatial mesh sizes are reduced by $50 \%$ in one direction, and later in both directions, and the results are compared. It is observed that, when the mesh size is reduced by $50 \%$ in the $y$-direction, the results differ in the fifth decimal place while the mesh sizes are reduced by $50 \%$ in $x$-direction or in both directions; the results are comparable to three decimal places.

Hence, the above mesh sizes have been considered as appropriate for calculation. The coefficients $u_{i, j}^{n}$ and $v_{i, j}^{n}$ appearing in the finite-difference equations are treated as constants in any one time step. Here $i$-designates the grid point along the $x$-direction, $j$ along the $y$-direction. The values of $u, v$ and $\theta$ are known at all grid points at $t=0$ from the initial conditions.

The computations of $u, v, \theta$ and $\phi$ at time level $(n+1)$ using the values at previous time level $(n)$ are carried out as follows: The finite difference equation (21) at every internal nodal point on a particular $i-$ level constitutes a tridiagonal system of equations. Such systems of equations are solved by using Thomas algorithm as discussed in Carnahan et al. [22]. Thus, the values of $\phi$ are found at every nodal point for a particular $i$ at $(n+1)$ th time level. Similarly, the values of $\theta$ are calculated from Equation (20). Using the values of $\phi$ and $\theta$ at $(n+1)$ th time level in the equation (19), the values of $u$ at $(n+1)$ th time level are found in a similar manner. Thus, the values of $\phi, \theta$ and $u$ are known on a particular $i$-level. Finally, the values of $v$ are calculated explicitly using the Equation $(18)$ at every nodal point on a particular $i$-level at $(n+1)$ th time level. This process is repeated for various $i$-level. Thus the values of $\phi, \theta, u$ and $v$ are known, at all grid points in the rectangular region at $(n+1)$ th time level. 
In a similar manner, computations are carried out by moving along the $i$-direction. After computing values corresponding to each $i$ at a time level, the values at the next time level are determined in a similar manner. Computations are repeated until the steady-state is reached. The steady state solution is assumed to have been reached, when the absolute difference between the values of $u$, as well as temperature $\theta$ and concentration $\phi$ at two consecutive time steps are less than $10^{-5}$ at all grid points.

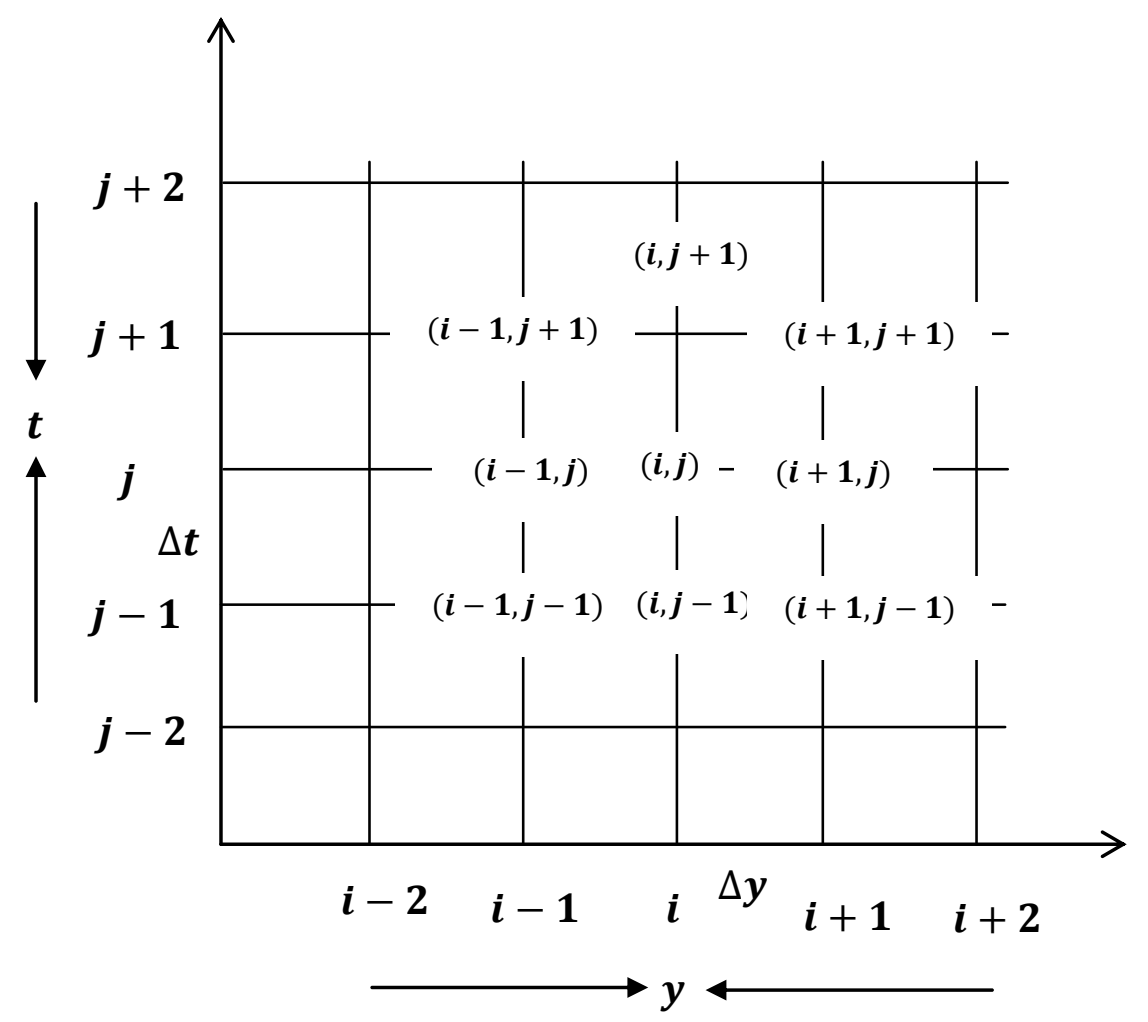

Fig. 2: Finite difference space grid

\section{Stability Analysis:}

The stability criterion of the finite difference scheme for constant mesh sizes are examined using Von-Neumann technique as explained by Carnahan et al. [22]. The general term of the Fourier expansion for $u, \theta$ and $\phi$ at a time arbitrarily called $t=0$, are assumed to be of the form $\exp (i \alpha x) \exp (i \beta y)$ (here $i=\sqrt{-1})$. At a later time $t$, these terms will become,

$\left.\begin{array}{rl}u & =F(t) \exp (i \alpha x) \exp (i \beta y) \\ \theta & =G(t) \exp (i \alpha x) \exp (i \beta y) \\ \phi & =H(t) \exp (i \alpha x) \exp (i \beta y)\end{array}\right\}$

Substituting Equations (22) in Equations (19) to (21) under the assumption that the coefficients $u, \theta$ and $\phi$ are constants over any one time step and denoting the values after one time step by $F^{\prime}, G^{\prime}$ and $H^{\prime}$. After simplification, we get

$$
\begin{aligned}
& \frac{F^{\prime}-F}{\Delta t}+\frac{u\left(F^{\prime}-F\right)[1-\exp (-i \alpha \Delta x)]}{2 \Delta x}+\frac{v\left(F^{\prime}+F\right) i \sin (\beta \Delta y)}{2 \Delta y} \\
& =\frac{\left(G^{\prime}+G\right) G r+\left(H^{\prime}+H\right) G r_{m}}{2}+\left(F^{\prime}+F\right)\left[\frac{\{\cos (\beta \Delta y)-1\}}{(\Delta y)^{2}}-\frac{\left(M+K^{-1}\right)}{2}\right] \\
& \frac{G^{\prime}-G}{\Delta t}+\frac{u\left(G^{\prime}-G\right)[1-\exp (-i \alpha \Delta x)]}{2 \Delta x}+\frac{v\left(G^{\prime}+G\right) i \sin (\beta \Delta y)}{2 \Delta y}
\end{aligned}
$$




$$
\begin{aligned}
& =\frac{\left(G^{\prime}+G\right)[\cos (\beta \Delta y)-1]}{\operatorname{Pr}(\Delta y)^{2}}-\frac{R_{a}\left(G^{\prime}+G\right)}{2 \operatorname{Pr}} \\
& \frac{H^{\prime}-H}{\Delta t}+\frac{u\left(H^{\prime}-H\right)[1-\exp (-i \alpha \Delta x)]}{2 \Delta x}+\frac{v\left(H^{\prime}+H\right) i \sin (\beta \Delta y)}{2 \Delta y} \\
& =\frac{\left(H^{\prime}+H\right)[\cos (\beta \Delta y)-1]}{S c(\Delta y)^{2}}-\frac{C_{r}\left(H^{\prime}+H\right)}{2}
\end{aligned}
$$

Equations (23) to (25) can be rewritten as,

$$
\begin{aligned}
& (1+A) F^{\prime}=(1-A) F+\frac{\Delta t}{2}\left[\operatorname{Gr}\left(G^{\prime}+G\right)+G r_{m}\left(H^{\prime}+H\right)\right] \\
& (1+B) G^{\prime}=(1-B) G \\
& (1+E) H^{\prime}=(1-E) H
\end{aligned}
$$

where

$$
\begin{aligned}
& A=\frac{u}{2} \frac{\Delta t}{\Delta x}[1-\exp (-i \alpha \Delta x)]+\frac{v}{2} \frac{\Delta t}{\Delta y} i \sin (\beta \Delta y)-[\cos (\beta \Delta y)-1] \frac{\Delta t}{(\Delta y)^{2}}+\frac{\left(M+K^{-1}\right) \Delta t}{2} \\
& B=\frac{u}{2} \frac{\Delta t}{\Delta x}[1-\exp (-i \alpha \Delta x)]+\frac{v}{2} \frac{\Delta t}{\Delta y} i \sin (\beta \Delta y)-[\cos (\beta \Delta y)-1] \frac{\Delta t}{\operatorname{Pr}(\Delta y)^{2}}+\frac{R_{a} \Delta t}{2 \operatorname{Pr}} \\
& E=\frac{u}{2} \frac{\Delta t}{\Delta x}[1-\exp (-i \alpha \Delta x)]+\frac{v}{2} \frac{\Delta t}{\Delta y} i \sin (\beta \Delta y)-[\cos (\beta \Delta y)-1] \frac{\Delta t}{S c(\Delta y)^{2}}+\frac{C_{r} \Delta t}{2 S c}
\end{aligned}
$$

After eliminating $G^{\prime}$ and $H^{\prime}$ in Equation (26) using Equations (27) and (28), the resultant equation is given by,

$$
(1+A) F^{\prime}=(1-A) F+G \frac{G r \Delta t}{(1+B)}+H \frac{G r_{m} \Delta t}{(1+E)}
$$

Equations (26) to (28) can be written in matrix form as follows:

$$
\left(\begin{array}{l}
F^{\prime} \\
G^{\prime} \\
H^{\prime}
\end{array}\right)=\left(\begin{array}{ccc}
\frac{1-A}{1+A} & D_{1} & D_{2} \\
0 & \frac{1-B}{1+B} & 0 \\
0 & 0 & \frac{1-E}{1+E}
\end{array}\right)\left(\begin{array}{l}
F \\
G \\
H
\end{array}\right)
$$

Where $D_{1}=\frac{G r \Delta t}{(1+A)(1+B)}$ and $D_{2}=\frac{G r_{m} \Delta t}{(1+A)(1+E)}$

Now, for stability of the finite difference scheme, the modulus of each Eigen value of the amplification matrix does not exceed unity. Since the matrix Equation (30) is triangular, the Eigen values are its diagonal elements. The Eigen values of the amplification matrix are $(1-A) /(1+A),(1-B) /(1+B)$ and $(1-E) /(1+E)$.

Assuming that, $u$ is everywhere non-negative and $v$ is everywhere non-positive, we get

$$
\begin{aligned}
& A=2 a_{1} \sin ^{2}\left(\frac{\alpha \Delta x}{2}\right)+2 c_{1} \sin ^{2}\left(\frac{\beta \Delta y}{2}\right)+i\left[a_{1} \sin (\alpha \Delta x)-b_{1} \sin (\beta \Delta y)\right]+\frac{\left(M+K^{-1}\right) \Delta t}{2} \\
& a_{1}=\frac{u}{2} \frac{\Delta t}{\Delta x}, \quad b_{1}=\frac{v}{2} \frac{\Delta t}{\Delta y}, \quad c_{1}=\frac{\Delta t}{(\Delta y)^{2}}
\end{aligned}
$$

Since the real part of $A$ is greater than or equal to zero, $|(1-A) /(1+A)| \leq 1$ always. Similarly, $\mid(1-$ $B) /(1+B) \mid \leq 1$ and $|(1-E) /(1+E)| \leq 1$. Hence, the finite difference scheme is unconditionally stable. The local truncation error is $O\left(\Delta t^{2}+\Delta y^{2}+\Delta x\right)$ and it tends to zero as $\Delta t, \Delta x$ and $\Delta y$ tend to zero. Hence, the scheme is compatible. Stability and compatibility ensures convergence.

\section{Results and Discussion}

Selected computations have been depicted graphically in all the figures. These figures generally show the spatial variable distribution at a variable time $t$. All parametric values corresponding to each figure are included 
therein. It should be noted that $G m=G r=5$ imply strong species and thermal buoyancy forces; $S c=0.60$ approximately simulates lower molecular weight gasses diffusing in air, $\operatorname{Pr}=0.71$ which represents air unless otherwise stated.

Table 1: Comparison of values of the flow velocity $(u)$ with those obtained by Loganathan et al. (2008) for $R_{a}=1.5, K=0.5, S c=0.78, m=n=0.7, t=1.75$ and $\operatorname{Pr}=0.71$ :

\begin{tabular}{|c|l|l|l|l|l|l|}
\hline & \multicolumn{3}{|c|}{ Loganathan et al. (2008) } & \multicolumn{3}{c|}{ Present work } \\
\hline & \multicolumn{3}{|c|}{$G r=G r_{m}$} & \multicolumn{3}{c|}{$G r_{m}$} \\
\hline $\mathrm{y}$ & 0.0 & 5.0 & 10.0 & 0.0 & 5.0 & 10.0 \\
\hline 0.0 & 1 & 1 & 1 & 1 & 1 & 1 \\
\hline 2.0 & 1.472185 & 1.717228 & 1.874410 & 1.471693 & 1.717183 & 1.874381 \\
\hline 4.0 & 0.146033 & 0.193744 & 0.248211 & 0.147580 & 0.193670 & 0.248146 \\
\hline 6.0 & 0.028831 & 0.052325 & 0.057653 & 0.028706 & 0.052321 & 0.057638 \\
\hline 8.0 & 0.004672 & 0.012958 & 0.012920 & 0.004661 & 0.012943 & 0.012905 \\
\hline 10.0 & 0.000729 & 0.003152 & 0.002830 & 0.000728 & 0.003147 & 0.002814 \\
\hline
\end{tabular}

In order to ascertain the accuracy of the numerical results, the results for the present study are compared with the available exact solution in the literature. The velocity profiles for $R_{a}=1.5, K=0.5, S c=0.60, m=n=$ $0.7, t=1.75$ and $\operatorname{Pr}=0.71$ are compared with the available exact solution of Loganathan et al. (2008) at $t=0.2$ in Table 1 . It is clearly observed from this table that the present results are in good agreement with the available theoretical solution at lower time level. This favorable comparison lends confidence in the numerical results reported subsequently.

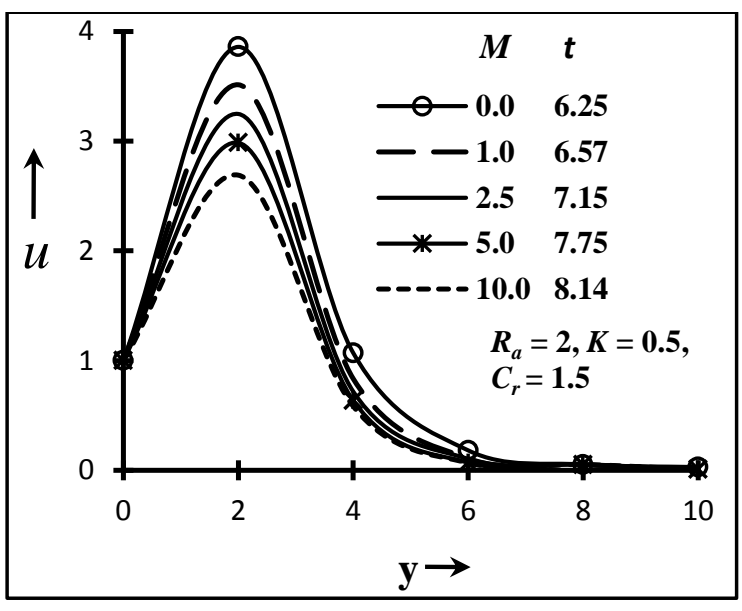

Fig. 1 (a): Steady state velocity profiles at $x=1.0$ for different $M$ and $t$

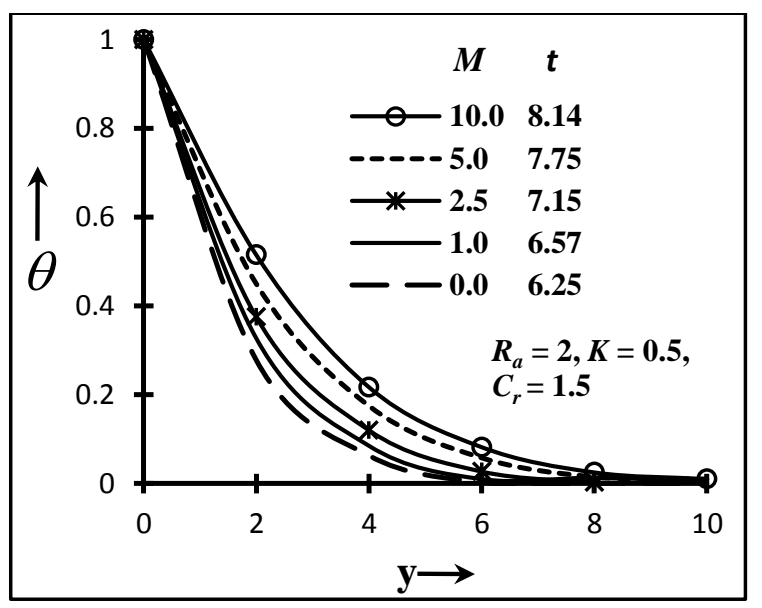

Fig. 1 (b): Steady state temperature profiles at $x=1.0$ for different $M$ and $t$

Fig. 1(a) to 1(c) present the response of velocity $(u)$, temperature $(\theta)$ and concentration $(\phi)$ to magnetohydrodynamic body force parameter $(M)$. Here $M=\left(\sigma B_{0}^{2} v\right) /\left(\rho u_{0}^{2}\right)$ and it signifies the ratio of Lorentz hydromagnetic body force to viscous hydrodynamic force. Increasing $M$ from 0 (non-conducting case), to 1.0 (magnetic body force and viscous force equal), 2.5, 5.0 through to 10.0 (very strong magnetic body force) induces a distinct reduction in velocities as shown in Fig. 1a. With higher $M$ values since the magnetic body force, $(-M u)$ in the momentum equation (11) is amplified, this serves to increasingly retard the flow. The imposition of an external magnetic field is therefore a powerful mechanism for inhibiting flow in the regime. The maximum velocities as before arise close to the surface of the plate, a short distance from it (at the surface, $y=0$ ); with further distance into the boundary layer, the profiles converge i.e. the magnetic body force has a weaker effect in the far field regime than in the near-field regime. Conversely with increasing $M$, temperature, (Fig. 1b) is observed to be markedly increased. This is physically explained by the fact that the extra work expended in dragging the fluid against the magnetic field is dissipated as thermal energy in the boundary layer, as elucidated by Sutton and Sherman (1965), Pai (1962) and Hughes and Young (1962). This results in heating of the boundary layer and an ascent in temperatures, an effect which is maximized some distance away from the surface. The magnetic field influence on temperatures while noticeable is considerably less dramatic than that 
on the velocity field, since the Lorentz body force only arises in the momentum equation (11) and influences the temperature $(\theta)$ and concentration $(\phi)$ fields, only via the thermal and buoyancy terms, Magnetic effects do not feature in either the temperature (12) or species diffusion equations (13). The deceleration in flow serves to enhance species diffusion in the regime and this causes a rise also in the concentration profiles (Fig. 1c) with increasing magnetic parameter. Applied external magnetic field, therefore while counteracting the momentum development in the regime, serves to enhance the heat and species diffusion, and this is of immense benefit in chemical engineering operations, where designers may wish to elevate transport in a regime without accelerating the flow.

Figs. 2(a) to 2(c) illustrate the influence of the porosity $(K)$ on the boundary layer variables, $u, \theta$ and $\phi$, respectively. Increasing the porosity of the porous medium clearly serves to enhance the flow velocity (Fig. 2a) i.e. accelerates the flow. This effect is accentuated close to the surface where a peak in the velocity profile arises. With further distance transverse to the surface, the velocity profiles are all found to decay into the free stream. An increased porosity clearly corresponds to a reduced presence of matrix fibers in the flow regime which, therefore, provides a lower resistance to the flow and in turn, boosts the momentum. For increasing $K$ values, the time $t$ required to attain the steady-state scenario is also elevated considerably. As such, the steady state is achieved faster for higher values of $K$. Conversely, with increasing porosity values, the temperature profile (Fig. 2b) in the regime is found to be decreases i.e. the boundary layer is cooled. A reduction in the volume of solid particles in the medium implies a lower contribution via thermal conduction. This serves to decrease the fluid temperature. As with the velocity field (Fig. 2a), the time required to attain the steady-state condition decreases substantially with a positive increase in $K$. In Fig. 2(c), a similar response for the concentration field is observed, as with the temperature distributions. The values of $\phi$ are continuously decreased with increasing values of porosity, also decreases with positive increases in the values of $K$, but reaches the steady state progressively faster.

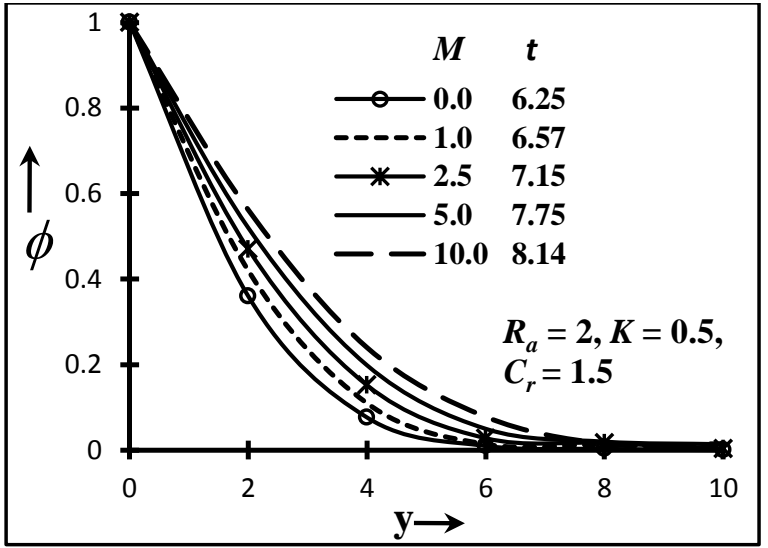

Fig. 1(c): Steady state concentration profiles at $x=1.0$ for different $M$ and $t$

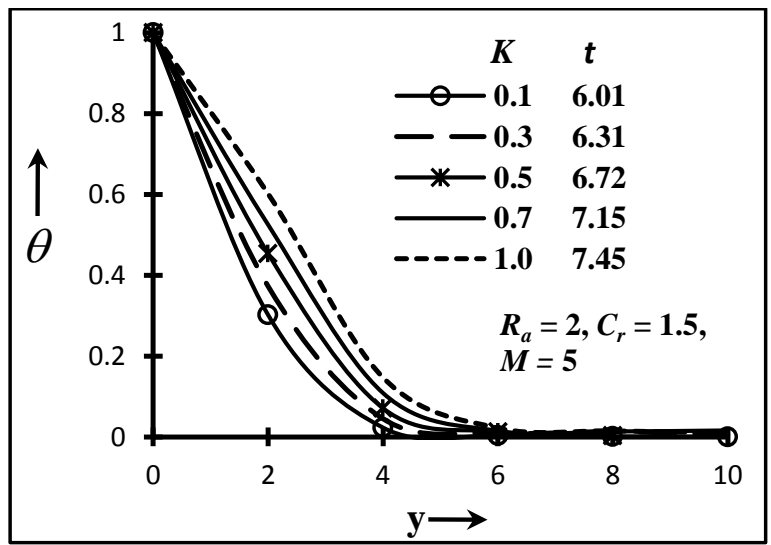

Fig. 2(b): Steady state temperature profiles at $x=1.0$ for different $K$ and $t$

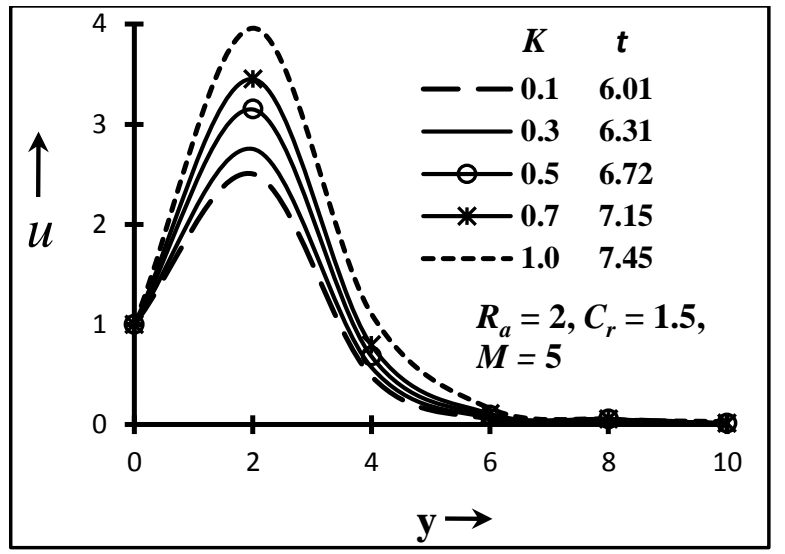

Fig. 2(a): Steady state velocity profiles at $x=1.0$ for different $K$ and $t$

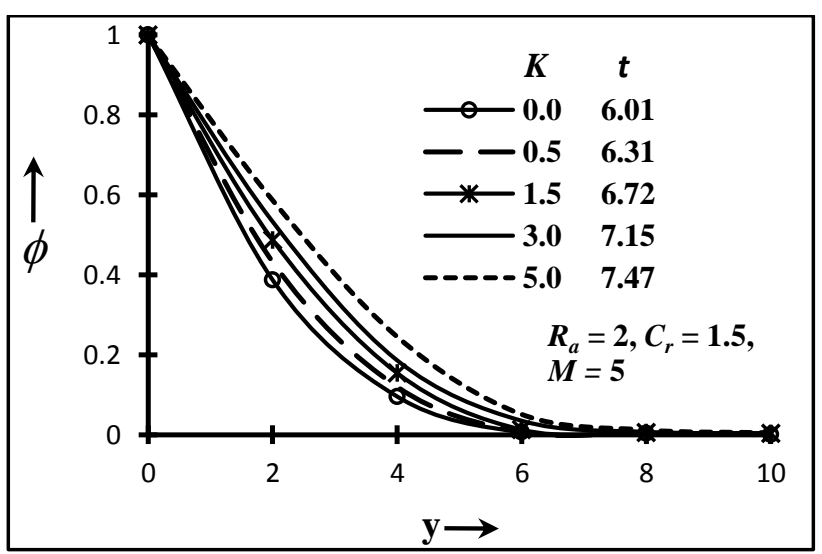

Fig. 2(c): Steady state concentration profiles at $x=1.0$ for different $K$ and $t$ 


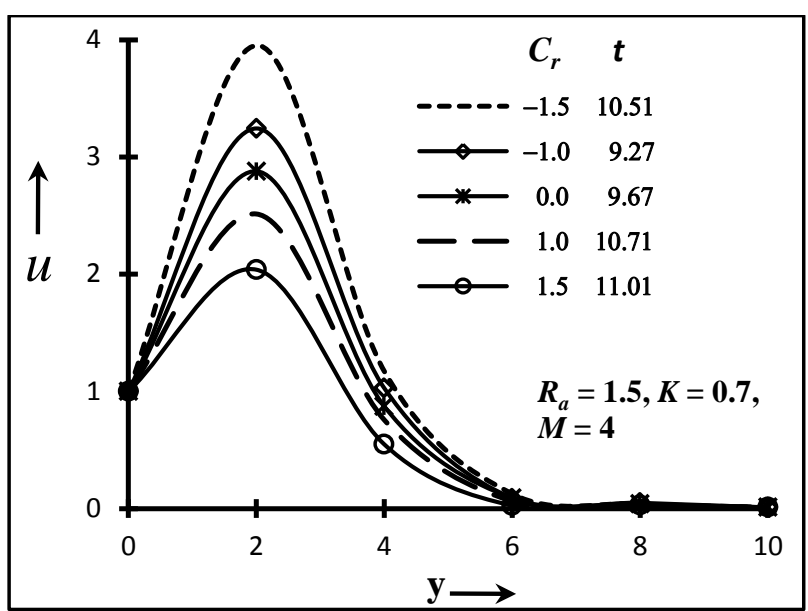

Fig. 3(a): Steady state velocity profiles at $x=1.0$ for different $C_{r}$ and $t$

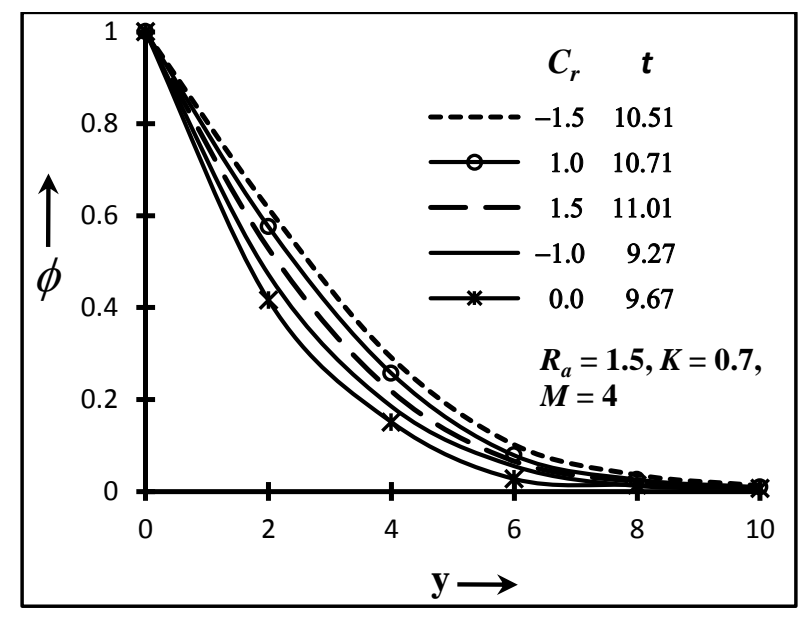

Fig. 3(c): Steady state concentration profiles at $x=1.0$ for different $C_{r}$ and $t$

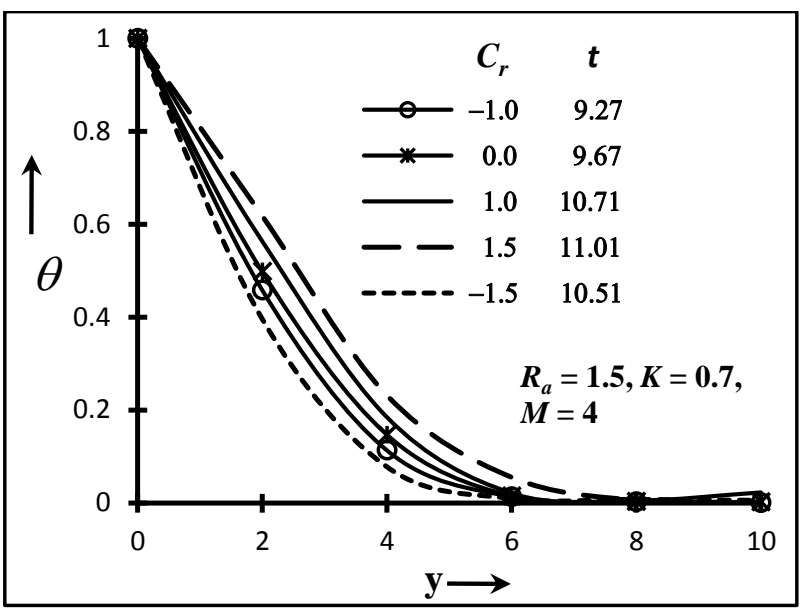

Fig. 3(b): Steady state temperature profiles at $x=1.0$ for different $C_{r}$ and $t$

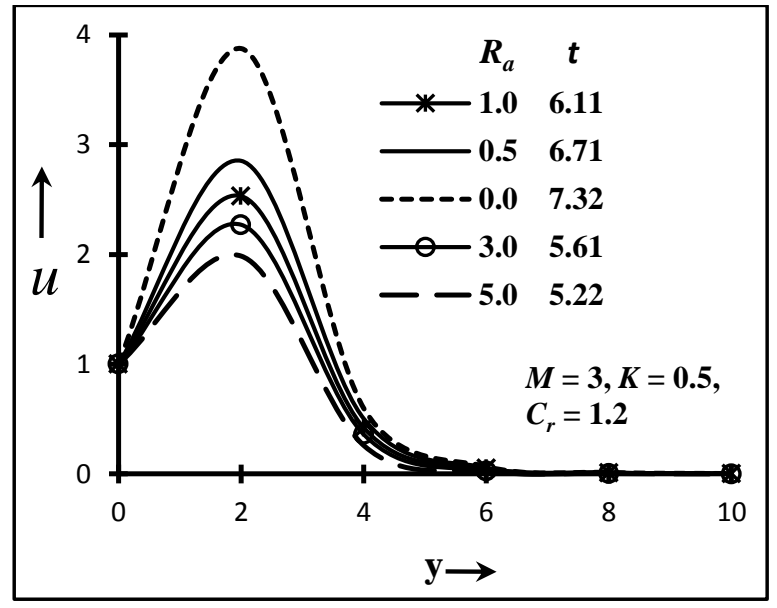

Fig. 4(a): Steady state velocity profiles at $x=1.0$ for different $R_{a}$ and $t$

Figs. 3(a) to 3(c) present the effects of the chemical reaction parameter, $C_{r}$, on the velocity $(u)$, temperature $(\theta)$ and concentration $(\phi)$ profiles, respectively. Physically, the mass diffusion Eq. (13) can be adjusted to represent a destructive chemical reaction (means endothermic, i.e., heat is absorbed) if $C_{r}>0$ or a generative chemical reaction (means exothermic, i.e., heat is generated) if $C_{r}<0$. Endothermic reactions cannot occur spontaneously, due to that a work must be done in order to get these reactions to occur. When endothermic reactions absorb energy, a temperature drop is measured during the reaction. Endothermic reactions are characterized by positive heat flow (into the reaction) and an increase in enthalpy. Exothermic reactions may occur spontaneously and result in higher randomness or entropy of the system. They are denoted by a negative heat flow (heat is lost to the surroundings) and decrease in enthalpy. There is a clear increase in the velocity values at the wall accompanying a rise in $C_{r}$ from -1.5 through $-1.0,0.0,1.0$ to 1.5 , i.e. the flow is accelerated throughout the boundary layer. This shows that velocity increases during generative reaction and decreases in destructive reaction. Irrespective of the values of $C_{r}$ or $t$, it is important to highlight that there is no flow reversal condition, i.e. no back flow in the boundary layer regime. The velocity $u$ sustains positive values throughout the flow regime. With an increase in the value of $C_{r}$, the time taken to attain the steady-state condition does not follow a direct increase or decrease. For $C_{r}=-1.5, t=10.51$ a value which decreases to 9.27 for $C_{r}=-1.0$ but then increases to 9.67 for $C_{r}=0.0$ and then continues to increase to 10.71 for $C_{r}=1.0$ and finally increases to 11.01 for $C_{r}=1.5$. The steady-state condition is therefore, achieved fastest for $C_{r}=-1.0$. Conversely, with an increase in the value of $C_{r}$, the temperature, $\theta$, as shown in Fig. $3 \mathrm{~b}$, increases continuously through the 
boundary layer. Again the steady-state condition is attained fastest for $C_{r}=-1.0$ but slowest for $C_{r}=1.5$. Fig. $3 \mathrm{c}$ indicates that a rise in the value of $C_{r}$ strongly suppresses the concentration levels in the boundary layer regime. All profiles decay monotonically from the surface (wall) to the free stream. The concentration boundary layer thickness is therefore, considerably greater for $C_{r}=-1.5$ than for $C_{r}=1.5$.

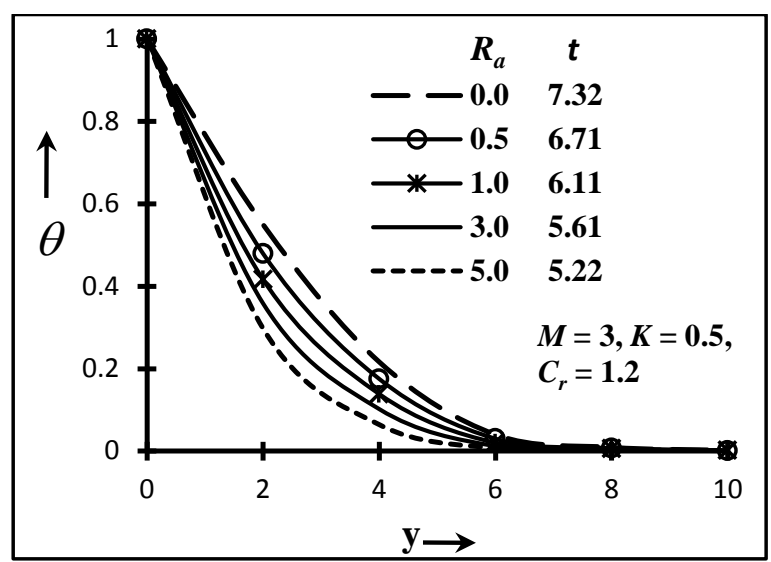

Fig. 4(b): Steady state temperature profiles at $x=1.0$ for different $R_{a}$ and $t$

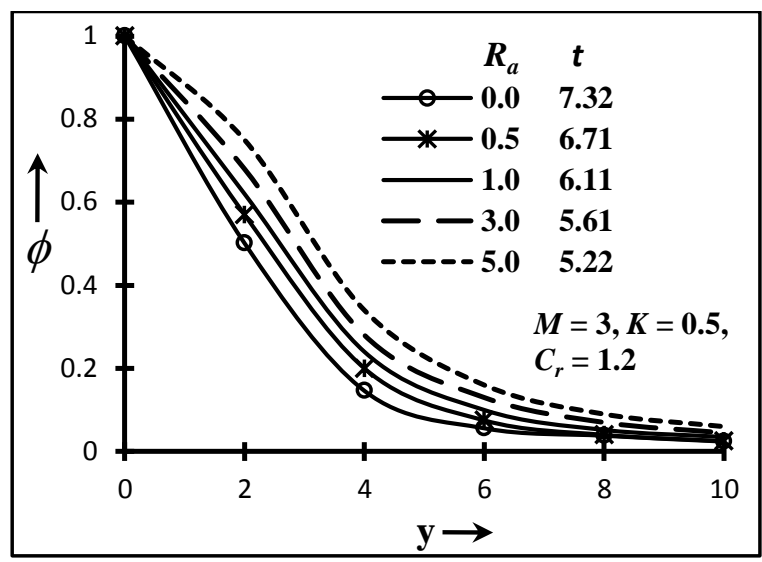

Fig. 4(c): Steady state concentration profiles at $x=1.0$ for different $R_{a}$ and $t$

In Figs. 4(a) to 4(c), the influence of the radiation parameter, $R_{a}$ on the steady-state velocity, temperature and concentration distributions with distance transverse to the surface (i.e. with $y$-coordinate) are presented, respectively. The parameter $R_{a}$ defines the ratio of thermal conduction contribution relative to thermal radiation. For $R_{a}=1$, the thermal radiation and the thermal conduction contributions are equivalent. For $R_{a}>1$, the thermal radiation effect is dominant over the thermal conduction effect and vice versa for $R_{a}<1$. An increase in the value of $R_{a}$ from 0 (non-radiating) through 0.5 (thermal conduction is dominant over radiation), 1.0, 3.0, 5.0 to 8.0 (radiation is dominant over thermal conduction), causes a significant decrease in the velocity with distance into the boundary layer i.e. decelerates the flow. The velocities in all cases ascend from the surface, peak close to the wall and then decay smoothly to zero in the free stream. It is also noted that with increasing values of the parameter $R_{a}$, the time taken to attain the steady state condition is reduced. Therefore, it is concluded that the thermal radiation flux has a de-stabilizing effect on the transient flow regime. This is important in polymeric and other industrial flow processes since it shows that the presence of thermal radiation while decreasing temperatures, will affect flow control from the surface into the boundary layer regime. As expected, the temperature values are also significantly reduced with an increase in the value of $R_{a}$ as there is a progressive decrease in the thermal radiation contribution accompanying this. All profiles show monotonic decays from the wall to the free stream. The maximum reduction in temperatures is witnessed relatively close to the surface since thermal conduction effects are prominent closer to the surface, rather than further into the free stream.

The concentration $(\phi)$ profile is conversely boosted with an increase in the value of $R_{a}$ (i.e. decrease in thermal radiation contribution). The parameter $R_{a}$ does not arise in the species conservation equation (13) and therefore, the concentration field is indirectly influenced by $R_{a}$ via the coupling of the energy equation (12) with the momentum equation (11), the latter also being coupled with the convective acceleration terms in the species equation (13). However, as with the response of the velocity and temperature fields, an increase in the value of $R_{a}$ decreases the time that elapses to achieve the steady-state condition. Therefore, while greater thermal radiation augments diffusion of species in the regime, it requires greater time to achieve the steady-state condition. 


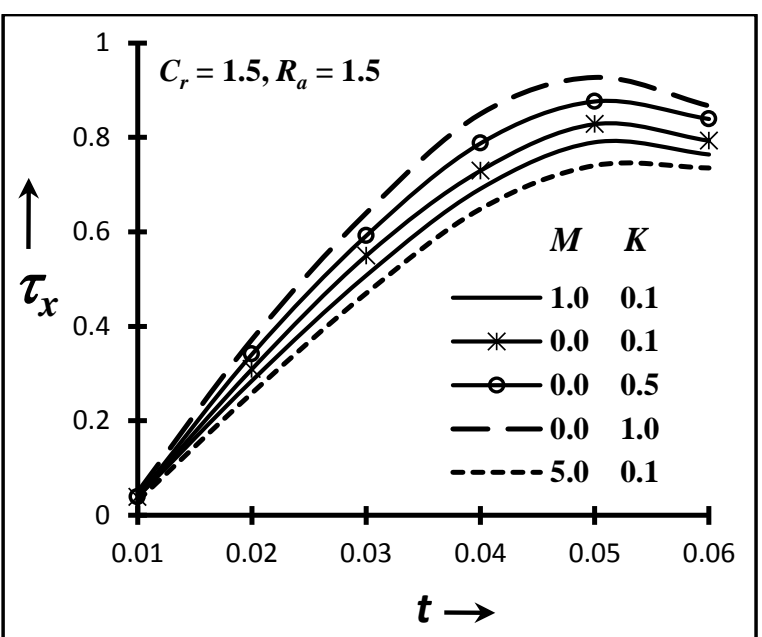

Fig. 5: Effects of $M$ and $K$ on local skin friction for different $t$

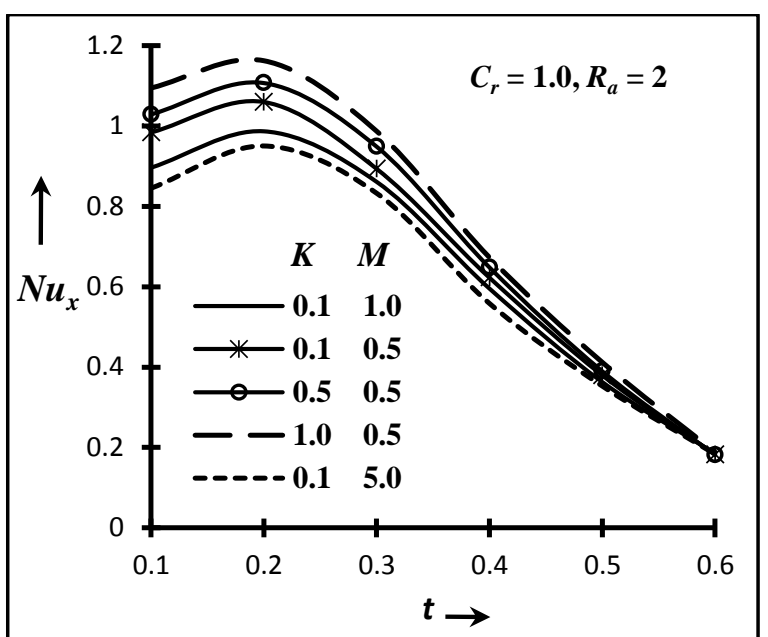

Fig. 6: Effects of $K$ and $M$ on local Nusselt number for different $t$

Fig. 5 show the effects of porosity $(K)$ and magnetic parameter $(M)$ on shear stress function $\left(-u^{\prime}\right)$ plotted against time variable $(t)$. Increasing the porosity parameter $(K)$ clearly enhances the skin friction since with progressively decreasing solid matrix fibers to resist the flow, the fluid will be accelerated and shear faster past the surface of the wall. However, it is interesting to note that for each profile, the shear skin friction initially grows from the lower value of time (here $t=0.01$, where it vanishes owing to the no-slip condition), peaks at $t=0.05$ and thereafter descends in value when $t>0.06$ (which although not included in the graph scale, clearly indicates that profiles are decaying towards it). Therefore, the maximum shear stress arises for any porosity value at the time $t=0.05$ point on the surface of the wall. The converse response is seen for $M$, wherein a sharp decrease in skin friction is induced by increasing the magnetic parameter, $M$ from 0 (electrically non-conducting case) through 1.0, to 5.0. Here $M=\left(\sigma B_{0}^{2} v\right) /\left(\rho u_{0}^{2}\right)$ and is directly proportional to the applied radial magnetic field, $B_{0}$, for constant electrical conductivity $(\sigma)$, fluid density $(\rho)$, kinematic viscosity $(v)$ and plate velocity $\left(u_{0}\right)$. Therefore greater retarding effect is generated in the flow with greater $M$ values (i.e. stronger magnetic field strengths) which causes the prominent depression in skin friction. For $M=1$, the magnetic drag force will be of the same order of magnitude as the viscous hydrodynamic force. For $M>1$, hydromagnetic drag will dominate and vice versa for $M<1$. In magnetic materials processing, the flow can therefore be very effectively controlled with a magnetic field.

Fig. 6 show the influence of porosity $(K)$ and magnetic parameter $(M)$ on surface heat transfer rate i.e. Nusselt number function $\left(-\theta^{\prime}\right)$ plotted against time variable $(t)$. An increase in porosity clearly enhances $\left(-\theta^{\prime}\right)$ values which are consistent with the reduction of temperatures in the boundary layer regime, computed earlier. The negative sign for $\left(-\theta^{\prime}\right)$ indicates that heat is being conducted away from the boundary layer to the surface with increasing porosity and this causes a simultaneous decrease in temperatures in the boundary layer i.e. cools the regime. Peak Nusselt number function is located consistently at the plate (i.e. at the smaller time) and in all cases, profiles decay smoothly to a minimum value (i.e. at the greater time). A contrary response is computed for the effect of magnetic parameter, $M$, on the Nusselt number function $\left(-\theta^{\prime}\right)$ distributions in same figure. Increasing magnetic field enhances the Lorentzian hydromagnetic drag which serves to decelerate the flow and warm the fluid. Therefore heat will be transferred at progressively lower rates from the fluid to the surface.

Finally in Fig. 7, the response of surface mass transfer rate i.e. Sherwood number function $\left(-\phi^{\prime}\right)$ to a change in porosity $(K)$ and magnetic parameter $(M)$, respectively, are shown. As with the Nusselt number function distributions, $\left(-\phi^{\prime}\right)$ is found to be strongly enhanced with increasing porosity, but reduced with an increase in magnetic parameter. Again, the peak surface mass transfer rates arise at the surface of the wall for smaller time and decrease continuously towards zero for $t>0.6$. 


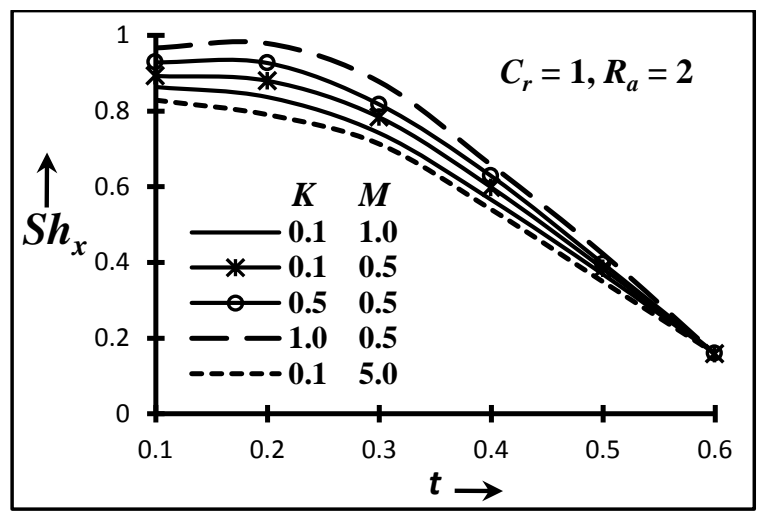

Fig. 7: Effects of $K$ and $M$ on local Nusselt number for different $t$

\section{Conclusions:}

In this paper, investigations were made to study the transient magneto-hydrodynamic convective heat and mass transfer in boundary layer flow from an impulsively-started and upward translating semi-infinite plate immersed in a porous medium in the presence of thermal radiation and homogeneous chemical reaction of first order. A flux model was employed to simulate thermal radiation effects. The dimensionless governing coupled, nonlinear partial differential equations for this investigation were solved by an efficient, accurate, and extensively validated and unconditionally stable finite-difference scheme of the Crank-Nicolson type. Some brief results of our investigation are mentioned as follows:

- It has been shown that increasing magnetic field generally decelerates the flow, but increases temperatures and concentration values in the regime.

- Increasing porosity serves to accelerate the flow but reduce temperatures and concentration values.

- The analysis showed that increasing the thermal radiation effects decelerated the flow velocity as well as the fluid temperature, but depressed the solute concentration.

- Increasing the porosity parameter boosted the wall shear stress as well as the Nusselt and Sherwood numbers, whereas increasing the magnetic parameter effectively reduced the shear stress as well as the Nusselt and Sherwood numbers at the wall.

- The present study was confined to Newtonian fluids. It is hoped that the present results be used as a vehicle for understanding more complex problems dealing with viscoelastic and power-law rheological fluid models.

\section{References}

Nield, D. A., and Bejan, A. (1999): Convection in Porous Media, Springer, New York. http://dx.doi.org/10.1007/978-1-4757-3033-3

Ingham, D. B., and Pop, I. (1998): Transport Phenomena in Porous Media, Pergamon, Oxford.

England, W. G., and Emery, A. F. (1969): Thermal radiation effects on the laminar free convection boundary layer of an absorbing gas, J. of Heat Transfer, Vol. 91, pp. 37-44.

Raptis, A., and Perdikis, C. (2003): Thermal radiation of an optically thin gray gas, Int. J. Applied Mechanical Engg., Vol. 8, No. 1, pp. 131-134.

Raptis, A. (1998): Radiation and free convection flow through a porous medium, International Communications in Heat and Mass Transfer, Vol. 25, No. 2, pp. 289-295. http://dx.doi.org/10.1016/S0735-1933(98)00016-5

Hussain, Md. A., and Pop, I. (2001): Radiation effects on free convection over a vertical flat plate embedded in a porous medium with high porosity, International Journal of Thermal Science, Vol. 40, No. 2, pp. 153-163.

Jaiswal, B. S., and Soundalgekar, V. M. (2001): Oscillating plate temperature effects on a flow past an infinite vertical porous late with constant suction and embedded in a porous medium, Heat and Mass Transfer, Vol. 37, pp. 125-131. http://dx.doi.org/10.1007/s002310000180 
Bakier, A. Y. (2001): Thermal radiation effect of mixed convection from vertical surfaces in saturated porous media. International Communications of Heat and Mass Transfer, 28 (1), 119-126. http://dx.doi.org/10.1016/S0735-1933(01)00219-6

Raptis, A., and Perdikis, C. (2004): Unsteady flow through a highly porous medium in the presence of radiation, Transport in Porous Media, Vol. 57, No. 2, pp. http://dx.doi.org/10.1023/B:TIPM.0000038262.65594.e8

Zueco, J. (2008): Unsteady free convection-radiation flow over a vertical wall embedded in a porous medium, Communications in Numerical Methods in Engineering, Vol. 24, No. 11, pp. 1093-1105. http://dx.doi.org/10.1002/cnm.1016

Mahmoud, A. A., and Chamkha, A. J. (2010): Non-similar solutions for heat and mass transfer from a surface embedded in a porous medium for two prescribed thermal and solutal boundary conditions, International Journal of Chemical Reactor Engineering, Vol. 8, pp. 1-24.

Sahin, A. (2008): Transient three dimensional flow through a porous medium with transverse permeability oscillating with time, Emirate Journal for Engineering research, Vol.13, No. 3, pp. 11-17.

Sahin, A. (2010): Free convective transient three-dimensional flow through a porous medium oscillating with time in presence of periodic suction velocity, International Journal of Applied Mathematics and Mechanics, Vol. 6, No. 11, pp. 1-16.

Chamkha, A. J. (2003): MHD flow of a uniformly stretched vertical permeable surface in the presence of heat generation/absorption and a chemical reaction, Int. Communication in Heat and Mass Transfer, Vol. 30, No. 3, pp. 413-422. http://dx.doi.org/10.1016/S0735-1933(03)00059-9

Muthucumaraswamy, R., and Kulaivel, T. (2003): Chemical reaction effects on moving infinite vertical plate with uniform heat flux and variable mass diffusion, Forschung im-Ingenieurwesen, Vo. 68, pp. 101-104. http://dx.doi.org/10.1007/s10010-003-0112-9

Sahin, A., and Chamkha, A. J. (2010): Effects of chemical reaction, heat and mass transfer and radiation on the MHD flow along a vertical porous wall in the presence of induced magnetic field, Int. Journal of Industrial Mathematics, Vol. 2, No. 4, pp. 245-261.

Sahin, A. (2009): The study of heat and mass transfer on free convective three-dimensional unsteady flows over a porous vertical plate, Journal of Energy, Heat and Mass Transfer, Vol. 31, pp. 89-110.

Sahin, A., and Liu, I. C. (2010): Mixed convective three-dimensional heat and mass transfer flow with transversely periodic suction velocity, Int. J. Applied Mathematics and Mechanics, Vol. 6, No. 1, pp. 58-73.

Yang, L. K. (1992): Forced convection in a vertical pipe with combined buoyancy and radiation effects, International Communications in Heat and Mass Transfer, Vol. 19, No. 2, pp. 249-262. http://dx.doi.org/10.1016/0735-1933(92)90036-H

Makinde, O. D. (2005): Free convection flow with thermal radiation and mass transfer past a moving vertical porous plate, International Communications in Heat and Mass Transfer, Vol. 32, No. 10, pp. 1411-1419. http://dx.doi.org/10.1016/j.icheatmasstransfer.2005.07.005

Loganathan, P., Kulandaivel, T., and Muthucumaraswamy, R. (2008): First order chemical reaction on moving semi-infinite vertical plate in the presence of optically thin gray gas, Int. J. of Appl. Math. And Mech., Vol. 4, No. 5, pp. 26-41.

Carnahan, B., Luther, H. A., and Wilkes, J. O. (1969): Applied Numerical Methods, John Wily and Sons, New York.

Bég, O. A., Takhar, H. S., Chamkha, A. J., Filip, D., and Pop, I. (2003): Mixed radiation convection flow of an optically dense viscous fluid along a vertical surface in a non-Darcy geological porous system, Int. J. Applied Mechanics and Engineering, Vol. 8, No. 3, pp. 483-496.

Chamkha, A. J., Takhar, H. S., and Bég, O. A. (2004): Radiative free convective non-Newtonian fluid flow past a wedge embedded in a porous medium, Int. J. Fluid Mechanics Research, Vol. 31, No. 2, pp. 101-115. DOI: 10.1615/InterJFluidMechRes.v31.i2.10. http://dx.doi.org/10.1615/InterJFluidMechRes.v31.i2.10

Sutton, G. W., and Sherman, A. S. (1965): Engineering Magnetohydrodynamics, MacGraw-Hill, New York. 
Pai, S.I. (1962): Magnetogasdynamics and Plasma Dynamics, Springer, Berlin. http://dx.doi.org/10.1007/978-37091-8083-9

Hughes, W. F., and Young, F. J. (1966): The Electromagnetodynamics of Fluids, John Wiley, New York.

Sahin, A., Batin, A., and Chamkha, A. J. (2014): Finite difference approach in porous media transport modeling for magnetohydrodynamic unsteady flow over a vertical plate: Darcian model, Int. J. of Numerical Methods for Heat and Fluid Flow, Vol. 24 No. 5, pp. 1204-1223. http://dx.doi.org/10.1108/HFF-01-2013-0008

Sahin, A., Zueco, J., and López-Ochoa, L.M., (2014): Numerical Modeling of MHD Convective Heat and Mass Transfer in Presence of First-Order Chemical Reaction and Thermal Radiation, Chemical Engineering Communications, Vol. 201, pp. 419-436. http://dx.doi.org/10.1080/00986445.2013.775645

Zueco, J., Sahin, A., and López-Ochoa, L.M., (2014): Magneto-micropolar flow over a stretching surface embedded in a Darcian porous medium, Arabian Journal for Science and Engineering. http://dx.doi.org/10.1007/s13369-014-1175-7 